\title{
On The Natural Shift of A Resonance Frequency
}

ROBERT J. HARRACH

U.S. DEPARTMENT OF COMMERCE National Bureau of Standards 


\section{THE NATIONAL BUREAU OF STANDARDS}

The National Bureau of Standards ${ }^{1}$ provides measurement and technical information services essential to the efficiency and effectiveness of the work of the Nation's scientists and engineers. The Bureau serves also as a focal point in the Federal Government for assuring maximum application of the physical and engineering sciences to the advancement of technology in industry and commerce. To accomplish this mission, the Bureau is organized into three institutes covering broad program areas of research and services:

THE INSTITUTE FOR BASIC STANDARDS ... provides the central basis within the United States for a complete and consistent system of physical measurements, coordinates that system with the measurement systems of other nations, and furnishes essential services leading to accurate and uniform physical measurements throughout the Nation's scientific community, industry, and commerce. This Institute comprises a series of divisions, each serving a classical subject matter area:

-Applied Mathematics-Electricity-Metrology-Mechanics-Heat-Atomic Physics-Physical Chemistry-Radiation Physics-Laboratory Astrophysics ${ }^{2}$ - Radio Standards Laboratory, ${ }^{2}$ which includes Radio Standards Physics and Radio Standards Engineering-Office of Standard Reference Data.

THE INSTITUTE FOR MATERIALS RESEARCH . . . conducts materials research and provides associated materials services including mainly reference materials and data on the properties of materials. Beyond its direct interest to the Nation's scientists and engineers, this Institute yields services which are essential to the advancement of technology in industry and commerce. This Institute is organized primarily by technical fields:

-Analytical Chemistry-Metallurgy-Reactor Radiations-Polymers-Inorganic Materials-Cryogenics ${ }^{2}$ - Materials Evaluation Laboratory-Office of Standard Reference Materials.

THE INSTITUTE FOR APPLIED TECHNOLOGY ... provides technical services to promote the use of available technology and to facilitate technological innovation in industry and government. The principal elements of this Institute are:

-Building Research-Electronic Instrumentation-Textile and Apparel Technology CenterTechnical Analysis-Center for Computer Sciences and Technology-Office of Weights and Measures-Office of Engineering Standards Services-Office of Invention and Innovation-Clearing. house for Federal Scientific and Technical Information. ${ }^{3}$

${ }^{1}$ Headquarters and Laboratories at Gaithersburg, Maryland, unless otherwise noted; mailing address Washington, D. C., 20234.

2 Located at Boulder, Colorado, 80302.

${ }^{3}$ Located at 5285 Port Royal Road, Springfield, Virginia, 22151. 


\title{
UNITED STATES DEPARTMENT OF COMMERCE - John T. Connor, Secretary NATIONAL BUREAU OF STANDARDS • A. V. Astin, Director
}

\section{NBS TECHNICAL NOTE 346 \\ ISSUED SEPTEMBER 29, 1966}

\author{
ON THE NATURAL SHIFT OF A \\ RESONANCE FREQUENCY
}

\author{
ROBERT J. HARRACH \\ Radio Standards Laboratory \\ Institute for Basic Standards \\ National Bureau of Standards \\ Boulder, Colorado 80302
}

NBS Technical Notes are designed to supplement the Bureau's regular publications program. They provide a means for making available scientific data that are of transient or limited interest. Technical Notes may be listed or referred to in the open literature. 

1. ABSTRACT....................... v

2. INTRODUCTION. ....................... I

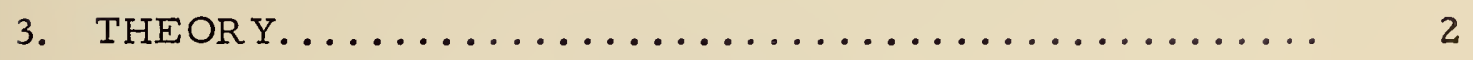

4. EXPERIMENT........................ 10

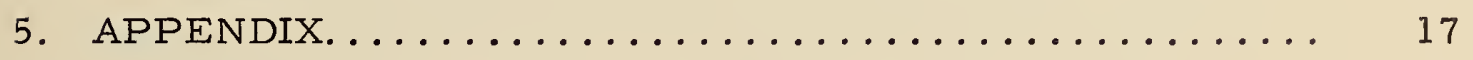

6. REFERENCES. ......................... 19

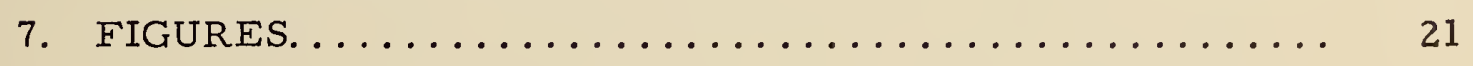





\author{
Robert J。Harrach \\ National Bureau of Standards, Boulder, Colorado
}

\begin{abstract}
The natural resonance frequency shift, caused by the transition-inducing radiation field, is examined for a magnetic dipole transition between hyperfine structure levels in the ground state of a thallium atom. A calculation predicts, for an atomic beam experiment, a natural shift magnitude of 1.4 parts in $10^{10}$ of the thallium resonance frequency, per $\mathrm{mW} / \mathrm{Oe}$. In the experiment, frequency shifts caused by overlap of neighboring resonances were observed, but the natural shift was unresolved, indicating that its size is more than an order of magnitude below the calculated value. Subsequently it has been shown that the natural shift was inhibited by the particular radiation field mode used in the experiment. When the theory correctly takes this mode into account, the calculated natural frequency shift is consistent with the experimental results.
\end{abstract}

Key Words: Atomic Beam, Frequency Shift, Radiation Field, Resonance, Thallium 



\section{INT RODU CTION}

The magnetic and electric components of a radiation field that induces transitions between a pair of stationary state energy levels, perturb the levels in a manner that depends on the radiation field intensity. Consequently the resonance frequency for the transition is a function of this intensity, exhibiting a "natural" shift away from the Bohr separation frequency of the two levels in the unperturbed atom.

In a calculation the natural shift arises from two contributions. One depends on the radiation field coupling neighboring energy levels to the pair involved in a given transition. A second contribution is due to the "anti-rotating" or non-resonant time component of the oscillating radiation field. The latter effect has as its leading term the familiar Bloch-Siegert expression, [1] and the former has been discussed by Salwen, [2] Ramsey, [3] Mizushima, [4] Shirley, [5] and others. [6]

In this paper a derivation is given of the natural shift for a fourlevel quantum system of the type displayed by the ground state hyperfine structure levels of a hydrogen or thallium atom in a weak external magnetic field. An attempt to measure this shift with an atomic beam experiment using thallium atoms and Rabi-type (single radiation field) excitation is then described. In studying a magnetic dipole $\pi$-transition between the levels $\left(F, M_{F}\right)=(1,1)$ and $\left(F, M_{F}\right)=(0,0)$, it was found that the natural shift was not resolved, indicating that its size is more than an order of magnitude below the calculated value. Subsequently, it has been shown by J. Shirley [17] that the natural shift was inhibited by the particular radiation field mode used in the experiment. Appreciable radiation field intensity-dependent shifts were observed for very small external magnetic field magnitudes (extreme proximity of neighboring energy levels), with the shifts becoming larger than 2 parts in 
$10^{9}$ of the resonance frequency value, per milliwatt variation of input intensity to the radiation field. These are attributed to ordinary overlap of the $(1,1) \leftrightarrow(0,0)$ transition with the weakly excited $(1,0) \leftrightarrow(0,0)$ transition.

In the Appendix consideration is given to the natural frequency shift of an atomic beam resonance when the excitation is made by a pair of separated radiation fields. Shift expressions are given for two transitions between hyperfine structure levels in the ground state of cesium-133.

\section{THEORY}

Interest will be confined to magnetic dipole interactions in quantum systems for which the Stark effect is negligible. The radiation field is then specified as an oscillating magnetic field, $\underset{\sim}{\mathrm{H}}(\mathrm{t})=\underset{\sim}{\mathrm{H}} \cos (\omega t)$, and the interaction between the quantum system and field is given by the semiclassical Hamiltonian $\hat{V}(t)=-\underset{\sim}{\mu} \cdot \underset{\sim}{H} \cos (\omega t)$, where $\underset{\sim}{\mu}$ is the magnetic dipole moment. Let $\hat{K}$ o be the Hamiltonian of the system in a weak external magnetic field, $\underset{\sim}{\mathrm{H}}$, with nondegenerate eignevalues $\mathrm{W}_{\mathrm{k}}$ corresponding to a complete orthonormal set of eigenfunctions $\left\{U_{k}(x)\right\}$ :

$$
\begin{gathered}
\hat{\mathcal{H} C} \underset{\mathrm{O}}{\mathrm{U}} \underset{\sim}{\sim}(\mathrm{x})=\mathrm{W}_{\mathrm{k}} \mathrm{U}_{\mathrm{k}} \underset{\sim}{\sim}(\mathrm{x}), \mathrm{W}_{\mathrm{k}+\mathrm{l}}>\mathrm{W}_{\mathrm{k}}, \\
\int \mathrm{U}_{\mathrm{j}}^{*} \underset{\sim}{(\mathrm{x})} \mathrm{U}_{\mathrm{k}}(\mathrm{x}) \mathrm{dx}=\delta_{\sim \mathrm{k}} .
\end{gathered}
$$

The problem is then to solve the Schrödinger equation:

$$
\left.i \hbar \frac{\partial}{\partial t} \underset{\sim}{(x}, t\right)=\left[\hat{H} \hat{H}_{0}+\hat{V}(t)\right] \underset{\sim}{\Psi}(x, t)
$$

If a solution is sought as an expansion in the eigenfunctions of He in the form

$$
\underset{\sim}{(\mathrm{x}, \mathrm{t})}=\sum_{\mathrm{k}} \mathrm{C}_{\mathrm{k}}(\mathrm{t}) \mathrm{e}^{-\mathrm{i} \xi} \mathrm{k}^{\mathrm{t}} \mathrm{U}_{\mathrm{k}} \underset{\sim}{(\mathrm{x})},
$$

then the Schrödinger equation is replaced by a set of coupled differential equations in the time-dependent amplitudes $C_{k}(t)$ : 
$i \frac{d}{d t} C_{j}(t)=\left[\omega_{j}-\xi_{j}\right] C_{j}(t)+e^{i\left(\omega+\xi_{j}\right) t} \sum_{k \neq j}^{\prime} b(j, k) e^{-i \xi_{k} t} C_{k}(t)$, all $j$,

where $w_{j} \equiv w_{j} / \hbar$, and

$$
2 b(j, k) \equiv-\frac{1}{\hbar} \int U \underset{j}{*} \underset{\sim}{*} \underset{\sim}{\sim} \cdot \underset{\sim}{H} U_{k}(\underset{d}{d} d \underset{\sim}{\sim} .
$$

To obtain Eq. (5) it has been assumed that $b(j, j)=0$, for all $j$, and the rotating field approximation has been used, replacing $\cos (\omega t)=\left(e^{i \omega t}+e^{-i \omega t}\right) / 2$ by the single exponential function $e^{i \omega t} / 2$. This neglect of the anti-rotating component of the radiation field precludes a derivation of the Bloch-Siegert shift in what follows.

The time-dependent part of the expansion (4) has been written in the form of J. Shirley's "phase factoring" transformation." [7] For many quantum systems it is possible, by making judicious choices for the values of the phase factors, $\left\{\xi_{k}\right\}$, to satisfy the set of equations

$$
w+\xi_{j}-\xi_{k}=0
$$

for all $j$ and $k$ for which $b(j, k) \neq 0 .[8]$ In this case, Eqs. (5) simplify to

$$
i \frac{d}{d t} C_{j}(t)=\left[w_{j}-\xi_{j}\right] C_{j}(t)+\sum_{k}^{b} b(j, k) C_{k}(t) \text {, all } j
$$

Eqs. (8) are amenable to a perturbation treatment, similar to that of [2] by which resonance frequency shifts may be calculated. 
A well known special case is that where the quantum system may be represented to a very good approximation as a two-level system, so that only two of the $C_{k}(t)$ in $(4)$, say $C_{p}(t)$ and $C_{q}(t)$, are nonzero. Then the choice of phase factors can be $[7]$

$$
\begin{aligned}
& \varepsilon_{p}=\left(\omega_{p}+\omega_{q}-\omega\right) / 2, \\
& \varepsilon_{q}=\left(\omega_{p}+\omega_{q}+\omega\right) / 2 .
\end{aligned}
$$

The pair of coupled differential equations, written as a single matrix equation, are then

$$
i \frac{d}{d t}\left(\begin{array}{l}
C_{p}^{(t)} \\
C_{q}(t)
\end{array}\right)=\left(\begin{array}{cc}
-\frac{1}{2}\left(\omega_{q p}-\omega\right) & b(p q) \\
b^{*}(p q) & +\frac{1}{2}\left(\omega_{q p}-\omega\right)
\end{array}\right)\left(\begin{array}{c}
C_{p}(t) \\
C_{q}(t)
\end{array}\right) .
$$

Eq. (10) can be solved exactly, and lead to the Rabi transition probability。 ${ }^{[9]}$ The resonance frequency for the transition in this case equals the Bohr frequency, $\omega_{q p} \equiv \omega_{q}-\omega_{p}$.

The result of bringing in energy levels other than the pair (p, q) involved in a given transition is to give, in a way to be described, an "effective" two-level problem, but with the elements of the $2 \times 2$ matrix Hamiltonian in Eq. (10) shifted to slightly different values. Thus consider the generalized two-level problem:

$$
i \frac{d}{d t}\left(\begin{array}{c}
C_{p}(t) \\
C_{q}(t)
\end{array}\right)=\left(\begin{array}{ll}
\alpha & b \\
b * & \beta
\end{array}\right)\left(\begin{array}{c}
C_{p}^{(t)} \\
C_{q}(t)
\end{array}\right)
$$


The general solution is

$$
\left(\begin{array}{c}
C_{p}(t) \\
C_{q}(t)
\end{array}\right)=a_{+}\left(t_{0}\right) e^{-i \lambda_{t}\left(t-t_{0}\right)}\left(\begin{array}{c}
\lambda_{p}(t) \\
\lambda_{q}(t)
\end{array}\right)+a_{-}\left(t_{0}\right) e^{-i \lambda_{-}\left(t-t_{0}\right)}\left(\begin{array}{c}
\lambda_{p}(-) \\
\lambda_{q}(-)
\end{array}\right)
$$

where the eigenvalues are (using the upper or lower sign throughout)

$$
\lambda_{ \pm}=\frac{(\alpha+\beta)}{2} \pm \frac{1}{2} \sqrt{(\alpha-\beta)^{2}+4|b|^{2}}
$$

and the corresponding orthonormal eigenvectors are

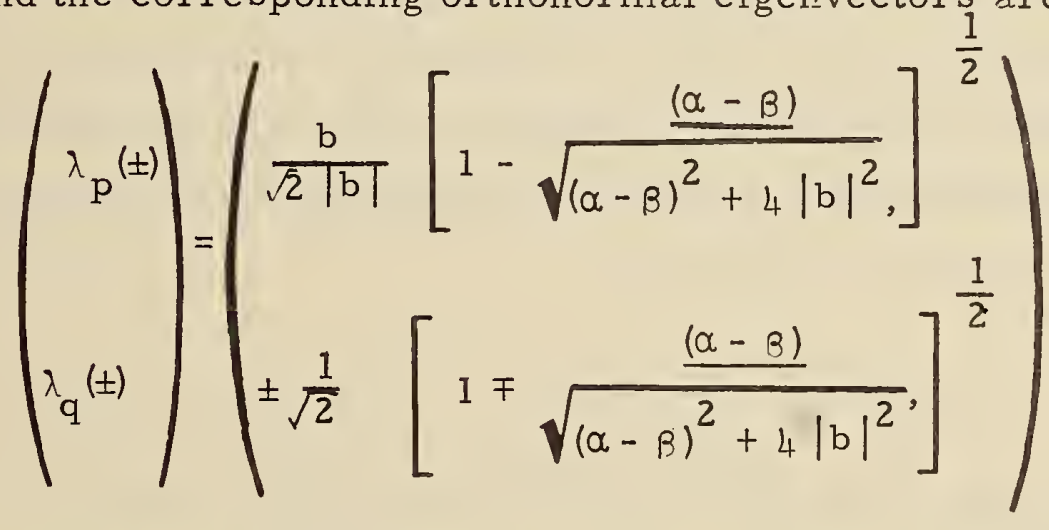

The constants $a_{ \pm}\left(t_{0}\right)$ are determined by the given initial state at $t=t_{0}$. The probability, $P$, that the quantum system makes a transition from its initial state after being exposed to the radiation field for a duration $T$ is defined in terms of the absolute value squared of the "overlap" of the initial state $\left(a t=t_{0}\right.$ ) onto the state vector at time $t_{0}+T:$

$$
\begin{aligned}
P & =1-\left|C_{p}^{*}\left(t_{0}\right) C_{p}\left(t_{0}+\tau\right)+C_{q}^{*}\left(t_{0}\right) C_{q}\left(t_{0}+\tau\right)\right|^{2} \\
& =4\left|a_{+}\left(t_{0}\right)\right|^{2}\left|a_{-}\left(t_{0}\right)\right|^{2} \sin ^{2}\left[\left(\lambda_{+}-\lambda_{-}\right) \tau / 2\right] .
\end{aligned}
$$


If the initial state is either $\mathrm{p}$ or $\mathrm{q}$, we find that the transition probability for an effective two-level system is

$$
P(p \leftrightarrow q)=\frac{4|b|^{2}}{\left[(\alpha-\beta)^{2}+4|b|^{2}\right.} \sin ^{2}\left[\frac{T}{2} \sqrt{(\alpha-\beta)^{2}+4|b|^{2}}\right] .
$$

For the special case of no neighboring energy levels, the matrix elements $\alpha, \beta$, and $b$ are given as in Eq. (10), and the Rabi transition probability results.

In treating an ensemble or beam of atoms interacting with the field, the sine-squared term in Eq. (16) is averaged over the ensemble. [10] The condition that defines the resonance frequency is that the denominator in $P(p \leftrightarrow q)$ take a minimum value. When $b$ is independent of frequency, this implies that the resonance condition is

$$
\alpha-\beta=0 \text {. }
$$

Evaluating $\alpha$ and $\beta$ for a given stationary state energy spectrum thereby determines the resonance frequency. This evaluation is now carried out for a specific example.

The ground state of a thallium (or hydrogen) atom in a weak external magnetic field, $\underset{\sim}{H}$, has four hyperfine structure levels, shown in Fig. 1, corresponding to the possible values of the total angular momentum quantum number, $F$, and the projection quantum number, $\mathrm{M}_{F^{\prime}}$, in the direction of $\mathrm{H}_{\sim} \cdot{ }^{[11]}$ Our interest will be in transitions from the single lower level, $\left(F, M_{F}\right)=(0,0)$, to one of the three relatively closely spaced upper levels. The frequencies of these transitions are in the microwave region.

In considering the effect on the $4 \leftrightarrow 1$ transition (ie, the $(1,1)$ $\hookrightarrow(0,0)$ transition) due to the levels 2 and 3 , we encounter four 
simultaneous differential equations of the form (5). The most significant radiation field couplings are represented (see Eq. (6)) by the matrix elements $b(1,4), b(1,3)$, and $b(1,2)$. The element $b(2,4)$ is identically zero by the magnetic dipole selection rule, $\Delta \mathrm{M}_{F}=0, \pm 1$. The elements $b(3,4)$ and $b(2,3)$ can be neglected as regards their influence on the $4 \leftrightarrow 1$ transition. [12]

Retaining only the three matrix elements, Eqs. (7) are satisfied by taking

$$
\begin{aligned}
& \xi_{1}=\left(w_{1}+w_{4}-w\right) / 2, \\
& \xi_{4}=\left(w_{1}+w_{4}+\omega\right) / 2=\xi_{3}=\xi_{2} .
\end{aligned}
$$

This produces four coupled equations of the form (8), that can be written as a single matrix equation containing the following $4 \times 4$ matrix Hamiltonian:

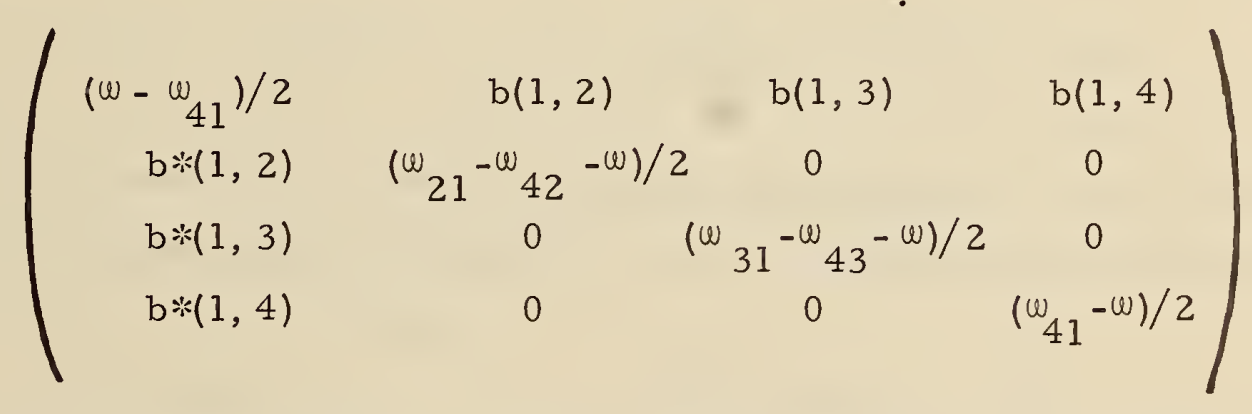

An iteration method is used to reduce this to a $2 \times 2$ matrix with diagonal elements $\alpha$, $\beta$ and off-diagonal elements $b, b *$. The procedure is to make the transformation $C_{k}(t)=\lambda_{k} e^{i \lambda t}, k=1,2,3,4$, in (8), thereby producing a $4 \times 4$ eigenvalue problem. The component equations are

$$
\begin{aligned}
& \lambda \lambda_{1} \equiv a \lambda_{1}+b \lambda_{4}=\left(\omega-w_{41}\right) \lambda_{1} / 2+b(1,2) \lambda_{2}+b(1,3) \lambda_{3}+b(1,4) \lambda_{4}, \\
& \lambda \lambda_{4} \equiv b * \lambda_{1}+\beta \lambda_{4}=b *(1,4) \lambda_{1}+\left(w_{41}-w\right) \lambda_{4} / 2, \\
& \lambda \lambda_{2}=b *(1,2) \lambda_{1}+\left(w_{21}-\omega_{42}-w\right) \lambda_{2} / 2, \\
& \lambda \lambda_{3}=b *(1,3) \lambda_{1}+\left(w_{31}-\omega_{43}-w\right) \lambda_{3} / 2 .
\end{aligned}
$$


The first two equations define $\alpha, \beta$, and $b$ for the effective two-level problem.

The zero-order approximation consists of taking $\lambda_{2}=\lambda_{3}=0$ (i.e., completely neglecting the neighboring energy levels). In this case, $\alpha=\left(\omega-\omega_{41}\right) / 2=-\beta$, and the resonance condition (17) gives $\omega_{\text {res }}=\omega_{41}$. The eigenvalue, by $\mathrm{Eq}$. (13), is

$$
\lambda= \pm \frac{1}{2} \sqrt{\left(w-w_{41}\right)^{2}+4|b(l, 4)|^{2}}
$$

In first-order and higher approximations, $\lambda_{2}$ and $\lambda_{3}$ in Eqs. (21) and (22) are solved in terms of $\lambda_{1}, \lambda_{4}, \lambda$, and $\omega$. In the se solutions $\lambda$ and $\omega$ are iteration parameters, denoted by $\lambda^{\prime}$ and $\omega^{\prime}$. We find that

$$
\begin{aligned}
& \lambda_{2}=-2 \lambda_{1} b *(1,2) /\left(\omega_{21}-\omega_{42}-\omega^{\prime}-2 \lambda^{\prime}\right), \\
& \lambda_{3}=-2 \lambda_{1} b *(1,3) /\left(\omega_{31}-\omega_{43}-\omega^{\prime}-2 \lambda^{\prime}\right) .
\end{aligned}
$$

Substituting these relations into (19) and (20) gives a pair of equations in $\lambda_{1}$ and $\lambda_{4}$ alone, and identifies $\alpha, \beta$, and $b$ for the problem as:

$\alpha=\left(\omega-w_{41}\right) / 2-\left[\frac{2|b(1,2)|^{2}}{\omega_{21}-w_{42}-\left(\omega^{\prime}+2 \lambda^{\prime}\right)}+\frac{2|b(1,3)|^{2}}{\omega_{31}-\omega_{43}-\left(\omega^{\prime}+2 \lambda^{\prime}\right)}\right]$,

$$
\begin{gathered}
B=\left(w_{41}-w\right) / 2, \\
b=b(1,4) .
\end{gathered}
$$

In $n$th - order, $w^{\prime}$ is taken to be the resonance frequency determined in order $(n-1)$, and $\lambda^{\prime}$ is the eigenvalue, Eq. (13), evaluated at $\omega^{\prime}$. [13] Thus, for first-order results, $w^{\prime}=w_{41}$ and $\lambda^{\prime}=|b(1,4)|$. The resonance 
condition (17) then gives the frequency shift:

$$
w_{\text {res }}-w_{41}=+\frac{2|b(1,2)|^{2}}{\left\langle w_{21}-w_{42}-w_{41}-2|b(1,4)|\right)}+\frac{2|b(1,3)|^{2}}{\left(w_{31}-w_{43}-w_{41}-2|b(1,4)|\right)} .
$$

Quantities like $|\mathrm{b}(\mathrm{j}, \mathrm{k})|^{2}$ are proportional to the square of the radiation field magnitude, and are thereby proportional to the radiation field intensity. Using the expressions of Torrey ${ }^{[14]}$, it is found for thallium that

$$
|b(1,4)|^{2} \cong \mu_{0}^{2} \mathrm{H}_{r}^{2} \sin ^{2}(\eta) / 72 \hbar^{2} \cong|b(1,2)|^{2},
$$

and

$$
|b(1,3)|^{2} \cong u_{0}^{2} H_{r}^{2} \cos ^{2}(\eta) / 36 \hbar^{2},
$$

where $u_{0}$ is the Bohr magneton, and $\mathrm{H}_{r} \sin (\eta)$ and $\mathrm{H}_{r} \cos (\eta)$ are, respectively, the components of the radiation field magnitude perpendicular to and parallel to the static magnetic field, $\underset{\sim}{\mathrm{H}}$.

The Bohr separation frequencies are evaluated using the BreitRabi formula ${ }^{[10]}$. For thallium,

$$
\begin{gathered}
w_{31} \cong w_{0}, w_{21} \cong w_{0}-x \mathrm{H}_{c^{\prime}}, w_{41} \cong w_{0}+x \mathrm{H}_{c^{\prime}} \\
w_{42} \cong+2 x \mathrm{H}_{c}, w_{43} \cong+x \mathrm{H}_{c^{\prime}}
\end{gathered}
$$

where $\omega_{0} / 2 \pi \equiv \nu_{0} \cong 2.13108 \times 10^{10} \mathrm{cps}$, and $\chi / 2 \pi \cong 4.65 \times 10^{5}$ (cps/Oe). 
Collecting these results, the first-order frequency shift expression for the $(I, I) \leftrightarrow(0,0)$ transition in thallium is

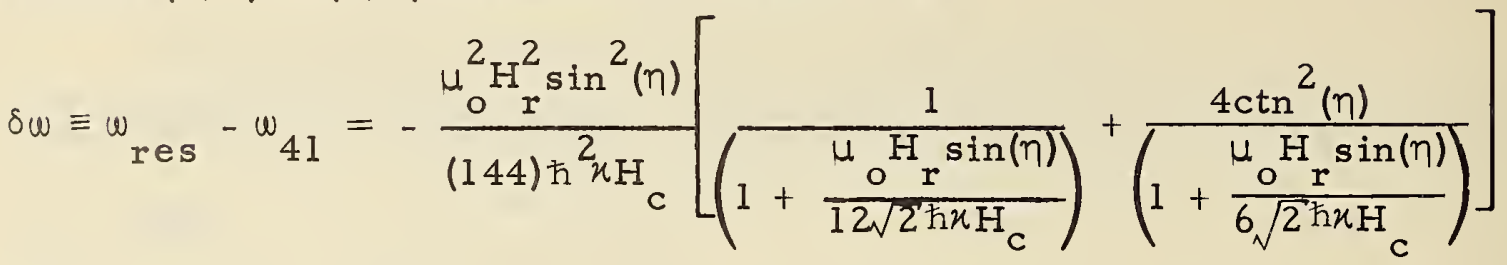

$$
\begin{aligned}
& \cong-\frac{\mu_{0}^{2} H_{r}^{2} \sin ^{2}(\eta)}{(144) \hbar^{2} \mu_{C}}\left[1+4 \operatorname{ctn}^{2}(\eta)\right] .
\end{aligned}
$$

The approximate form applies when the static field magnitude is large compared to the radiation field. The shift then varies linearly with radiation field intensity and inversely with the magnitude of the static magnetic field.

\section{EXPERIMENT}

An atomic beam experiment of the Rabi type, in which atoms traverse a single radiation field region, was performed to measure the natural frequency shift (29). A circular cylindrical, variable length cavity operated in a $\mathrm{TE}_{011}$ mode ${ }^{[15]}$ provides the radiation field. In this mode the important magnetic component oscillates parallel to the cavity axis, with a maximum amplitude directly on axis given by

$$
\mathrm{H}_{\mathbf{r}}(\mathrm{t})=\mathrm{H}_{\mathrm{r}} \sin \left(2 \pi \mathrm{x} / \lambda_{\mathrm{g}}\right) \cos (\omega t), \quad 0 \leq \mathrm{x} \leq \lambda_{\mathrm{g}} / 2,
$$

where $\lambda_{g}$ is the radiation wavelength in the cavity, and $x$ measures distance along the axis. Also present in this mode are a radial magnetic component and circumferential electric component, each of which has zero magnitude directly on axis. The radiation source is a phase-locked klystron, and power transmitted to the cavity through standard $\mathrm{K}$-band waveguide is measured by a bolometer. 
A beam of thallium-205 atoms effuses from an oven through a narrow rectangular slit and passes along the axis of the resonant cavity. The oven is operated at $900^{\circ} \mathrm{K}$, so that the most probable velocity ${ }^{[10]}$ for atoms in the beam is $\sqrt{3 / 2} \mathrm{v}_{\mathrm{O}} \equiv \sqrt{3 \mathrm{k}_{\mathrm{B}} \mathrm{T} / \mathrm{m}} \cong 3.4 \times 10^{4} \mathrm{~cm} / \mathrm{sec}_{\text {。 }}$ The resonant length of the cavity in vacuum $\left(4 \times 10^{-7}\right.$ torr $)$ is $2=16 \mathrm{~cm}$, giving a theoretical resonance line width ${ }^{[10]}$ of $(1.07) \mathrm{v}_{0} / \ell \cong 1.9 \mathrm{kcps}$ at optimum excitation intensity.

A constant magnetic field (c-field) is applied transverse to the beam by means of a current supplied to four rods in a rectangular array. The cavity is suspended into the center of the c-field, and the entire region is magnetically shielded. The polarizations of the applied c-field and oscillating magnetic field are mutually perpendicular, and magnetic dipole $\pi$-transitions are excited.

Deflection of the thallium atoms is made by two water-cooled inhomogeneous electromagnets at the entrance and exit to the shielded region. Each magnet has a 4-in. gap length, and gives a field magnitude and gradient at the beam of $6 \times 10^{3} \mathrm{Oe}$ and $2.3 \times 10^{4} \mathrm{Oe} / \mathrm{cm}$, respectively. Beyond the second inhomogeneous magnet, atoms which have made a transition converge on an oxidized tungsten wire detector, operated at a temperature of $1280^{\circ} \mathrm{K}$.

Measurement of the resonance frequency is made by a manual (as opposed to servo) technique. ${ }^{[16]}$ A single experiment consists of adjusting the $\mathrm{c}$-field current to a certain value and measuring the resonance frequency of the $(1,1) \leftrightarrow(0,0)$ transition for various levels of input power to the cavity.

Statistical uncertainties of the measured frequencies were typically 20 to $80 \mathrm{cps}$ (i.e., several parts in $10^{9}$ of the resonance frequency value). However, since the transition is strongly dependent on magnetic field, the measurement precision was limited by small, sporadic variations in the c-field. From the expression for $w_{41}$ in (28), it is seen that a change in 
c-field magnitude of only $1 \times 10^{-4}$ Oe results in a resonance frequency shift of almost $50 \mathrm{cps}$. Such a change can arise from a variation of about $0.1 \%$ in the c-field current, or from imperfect shielding of variations in the magnetic environment of the laboratory.

Within the uncertainty of the results, no radiation field intensitydependent frequency shift was observed for the $(1,1) \leftrightarrow(0,0)$ resonance until the c-field magnitude was reduced to a value slightly less than 0.050 Oe. Some representative data are shown in Figs. 2-4, where the input microwave power to the cavity is denoted by $\mathrm{p}$, and the experimentally determined optimum input power is $p_{0}=0.1 \mathrm{~mW}$. The frequency shifts are normalized to be zero at $\mathrm{p}=0$, and data are shown for values of $\mathrm{p} / \mathrm{p}_{\mathrm{o}}$ up to 240 .

The observed frequency shifts are fairly linear with $\mathrm{p}$, but show a definite "saturation" effect for small c-field magnitudes and high input powers. This tendency is very pronounced for the lowest $\mathrm{c}$-field value shown $(0.0108 \mathrm{Oe})$. For the other c-field magnitudes it is useful to find, by least squares analysis, the straight lines which best approximate the data. These appear as the solid lines in Figs. 2 and 3.

To interpret these results, we first evaluate the natural shift expression (29) for the atomic beam experiment. When the radiation field intensity is optimum for exciting an atomic beam resonance between any pair of levels $(j, k)$, the matrix element coupling these levels is given by $[10]$

$$
\left|\mathrm{b}_{\mathrm{o}}(\mathrm{j}, \mathrm{k})\right|=1.885 \mathrm{v}_{\mathrm{o}} / \mathrm{l}_{0}
$$

Using also the relations

$$
|b(j, k)|^{2} /\left|b_{o}(j, k)\right|^{2}=H_{r}^{2} / H_{r_{o}}^{2}=p / p_{o} \text {. }
$$


and Eqs. (26) and (27), we can express Eq. (29) as

$$
\delta \nu \equiv \frac{\delta w}{2 \pi} \cong-\frac{\left(1.885 v_{o} / \ell\right)^{2} p}{4 \pi x \mathrm{H}_{c} \mathrm{p}_{o}}\left[1+4 \operatorname{ctn}^{2}(\eta)\right] .
$$

With the appropriate values of $\mathrm{v}_{\mathrm{o}}, l$, and $\chi$, this becomes

$$
\delta_{\nu} \cong-\frac{0.30 p}{H_{c} p_{o}}\left[1+4 \operatorname{ctn}^{2}(\eta)\right] \text {, }
$$

where $\delta \nu$ is in cps and $\mathrm{H}_{c}$ in $\mathrm{Oe}$.

The applied c-field is perpendicular to the oscillating radiation field, but since the magnetic shielding is imperfect a residual static magnetic component exists in the apparatus, making the angle $\eta$ deviate somewhat from $\pi / 2$ radians. A lower limit for the magnitude of the natural shift is found by setting $\eta=\pi / 2$ in (33), and this lower limit is much larger than any shift observed. For example, at $\mathrm{H}_{c}=0.0469 \mathrm{Oe}$, the predicted shift is about $-60 \mathrm{cps} / \mathrm{mW}$, while that observed (Fig. 2 ) is about $-2 \mathrm{cps} / \mathrm{mW}$. At very low c-field magnitudes, the expression for the natural shift has to be carried to higher than first-order, and the shift is no longer linear but levels off at high intensity. This is in qualitative agreement with the data, but quantitatively the situation is not improved: the predicted natural shifts are more than an order of magnitude larger than any observed.

Part of this disagreement is accounted for in an "intuitive" way by considering the expression (30) for the transition-inducing magnetic field component. The field magnitude is not constant throughout the transition region, but varies as a half sine wave. This suggests that the $\mathrm{H}_{\mathrm{r}}^{2}$ term in (29) should be replaced by

$$
\mathrm{H}_{\mathrm{r}}^{2}\left\langle\sin ^{2}\left(2 \pi \times / \lambda_{g}\right)\right\rangle=\mathrm{H}_{\mathrm{r}}^{2} / 2 \text {. }
$$


This would reduce the natural shift by a factor of two. But, strictly speaking, the variation of the oscillating field magnitude makes the natural shift a function of atom velocity, and invalidates the simple resonance condition (17). Dr. J. Shirley ${ }^{[17]}$ has made a detailed analysis of the natural frequency shift which should result when the radiation field is of the form (30), and his results completely explain the depression of the natural shift magnitude below the level that could be clearly resolved in this experiment.

A study of other effects that could be responsible for the observed frequency shifts leads to the conclusion that overlap of the $(1,1) \leftrightarrow(0,0)$ transition with the $(1,0) \leftrightarrow(0,0)$ transition could be significant. The latter is a magnetic dipole $\sigma$-transition, excited by a component of the radiation field which is parallel to the static c-field. Because of imperfect magnetic shielding, such a component was present, and the $(1,0) \leftrightarrow(0,0)$ transition was weak but observable. The other thallium transition that could be important, the $(1,-1) \leftrightarrow(0,0)$ transition, was not observed, since atoms which make this transition are deflected away from the detector by the inhomogeneous magnets.

To calculate an expression for the overlap frequency shift, we can represent the line shapes by the Lorentzian function

$$
q(v)=q_{0} w^{2} /\left[4\left(v-v_{0}\right)^{2}+w^{2}\right]
$$

which gives a line centered at $v_{0}$, with maximum height $\mathrm{q}_{\mathrm{O}}$, and line width at half-maximum given by $w$. The variation of the line shape with radiation field intensity is fixed by determining experimentally the dependence of $\mathrm{w}$ and $\mathrm{q}_{\mathrm{O}}$ on the microwave input power, $\mathrm{p}$.

For two neighboring resonance lines of the form (34), it is easily found that the shift of the line center of one due to overlap with the other is given by

$$
\delta_{\nu} \cong\left(q_{0}^{\prime} / q_{0}\right)\left(w^{\prime}\right)^{2} w^{2} \Delta \nu /\left[4(\Delta v)^{2}+\left(w^{\prime}\right)^{2}\right]^{2},
$$


where $\Delta v$ is the separation of the unshifted line centers, and the prime refers to the neighboring line. From Eqs. (28), we have $\Delta v=-x \mathrm{H}_{\mathrm{c}} / 2 \pi$. The experimental determination of the input power dependence of the line width, shown in Fig. 5, gives

$$
\mathrm{w} \cong(2.2) 10^{3}\left(\mathrm{p} / \mathrm{p}_{\mathrm{o}}\right)^{1 / 4} \mathrm{cps}
$$

and $\mathrm{w}^{\prime}$ is given by the same relation. Thus the overlap frequency shift of the $(1,1) \leftrightarrow(0,0)$ resonance is expected to be

$$
\delta v \cong \frac{-(1.1) 10^{7}\left(\mathrm{q}_{\mathrm{o}}^{1} / \mathrm{q}_{\mathrm{o}}\right)\left(\mathrm{p} / \mathrm{p}_{\mathrm{o}}\right) \mathrm{H}_{\mathrm{c}}}{\left[(8.6) 10^{5} \mathrm{H}_{\mathrm{c}}^{2}+4.8\left(\mathrm{p} / \mathrm{p}_{\mathrm{o}}\right)^{1 / 2}\right]^{2}} \mathrm{cps}
$$

When the line separation is large, this simplifies to

$$
\delta v \cong-(1.5) 10^{-5}\left(q_{o}^{1} / q_{o}\right)\left(p / p_{o}\right) / H_{c}^{3} \mathrm{cps}
$$

The factor $\left(q_{0}^{\prime} / q_{0}\right)$ was found to be approximately $1 / 4$, though it varied slightly with both $\mathrm{p}$ and $\mathrm{H}_{\mathrm{c}}$.

Comparing the expressions (37) and (38) with the observed frequency shifts, it is found that they agree within an order of magnitude. Also the observed rates of frequency shift display the inverse cube dependence on c-field magnitude given in Eq. (38), as shown by the least squares determined line in Fig. 6. The observed shifts are therefore consistent with an interpretation in terms of overlap.

Despite the shortcomings of the experiment that has been described, two improvements would make it very well suited to an observation of the natural resonance frequency shift. First, one should use a radiation field mode in which the oscillating magnetic field component has constant amplitude 
over the length of the transition region (for a given instant in time), rather than a variable amplitude as given by Eq. (30). The natural shift would then have its full magnitude as given by Eqs. (29) and (32). Second, by using improved magnetic shielding and improved c-field geometry in the apparatus, the neighboring transition $\left(F, M_{F}\right)=(1,0) \leftrightarrow$ $\left(F, M_{F}\right)=(0,0)$ could be almost eliminated, and one would expect no sizeable frequency shifts due to overlap. The most significant effect that would compete with the natural shift would then probably be shifts due to unbalanced sidebands in the excitation spectrum. [3] The presence of such shifts could be ascertained by intentionally altering the sideband intensities 。 


\section{APPENDIX}

When an atomic beam experiment is of the Ramsey type,

$[10]$ employing excitation by a pair of radiation fields separated by a "drift" region, the natural frequency shift calculation is more complicated. The transition probability for the effective two-level problem in this case is found to be

$$
\begin{aligned}
& P(p \leftrightarrow q)=\frac{1}{4}\left(\frac{4|b|^{2}}{(\alpha-\beta)^{2}+4|b|^{2}}\right)\left|e^{-i \lambda}+^{\tau}-e^{-i \lambda}-\tau\right| \\
& x \mid \cos [(\bar{\omega} q \mathrm{qp}-\omega) \mathrm{T} / 2]\left(\mathrm{e}^{-\mathrm{i} \lambda}+^{\top}+\mathrm{e}^{-\mathrm{i} \lambda}-^{\top}\right) \\
& \left.+\frac{i(\alpha-\beta)}{\sqrt{(\alpha-\beta)^{2}+4|b|^{2}}} \sin [(\omega)-\omega) T / 2\right]\left.\left(e^{-i \lambda}+^{\top}-e^{-i \lambda}-{ }^{\top}\right)\right|^{2} \text {, }
\end{aligned}
$$

where $\bar{\omega}_{\text {qp }}$ is the space average Bohr frequency in the drift region of the apparatus, $\mathrm{T}=\mathrm{L} / \mathrm{v}$ is the time required by an atom with velocity $\mathrm{v}$ to traverse the drift region of length $L, \tau=\ell / v$ is the time required to traverse each of the radiation field regions of length $l, \lambda_{ \pm}$is given by Eq. (13), and $\alpha, \beta, b$ are defined by Eqs. (11). As before, $\alpha, \beta$, and $b$ are evaluated for a given stationary state energy level spectrum. A frequency increment $\delta \omega$ is defined, in a given order of the iteration calculation, by

$$
\alpha-\beta \equiv \omega-\omega_{\mathrm{qp}}-\delta \omega,
$$

where $w_{q p}$ is the Bohr frequency corresponding to the c-field magnitudes (assumed equal) in the two transition regions. But the resonance condition is no longer $\alpha-\beta=0$. Rather, 


$$
w_{\text {res }} \cong \bar{w}_{\mathrm{qp}}+\frac{\mathrm{kl}}{\mathrm{L}}\left(w_{\mathrm{qp}}+\delta w-\bar{w}_{\mathrm{qp}}\right) .
$$

The coefficient $k$ depends on the value of ( $\left.\omega_{q p}-\bar{w}_{q p}\right)$ and on the level of radiation field intensity. [18] For the "ideal" case where $\omega_{q p}=\bar{w}_{q p}, k$ is independent of intensity and approximately equals unity. The natural resonance frequency shift is then given by

$$
w_{\text {res }}-\bar{w}_{q p} \cong \frac{\ell}{I} \delta w .
$$

A special case of interest is the frequency standard transition between the $F=4, M_{F}=0$ and $F=3, M_{F}=0$ hyperfine structure levels of a cesium atom. Taking into account the nearest neighboring energy levels, shown in Fig. 7 , and retaining only the radiation field couplings indicated in the Figure, the natural frequency shift of the $(4,0) \leftrightarrow(3,0)$ resonance is derived to be

$$
\begin{aligned}
& \delta w_{\text {res }}=\left(w_{\text {res }}-\bar{w}_{52}\right) \\
& \cong \frac{2 l}{L}\left[\frac{\mid b\left(2,\left.6\right|^{2}\right.}{\left(w_{62}-w_{52}+w_{65}\right)}-\frac{|b(2,4)|^{2}}{\left(w_{52}-w_{42}+w_{54}\right)}+\frac{|b(1,5)|^{2}}{\left(w_{51}-w_{52}+w_{21}\right)}-\frac{|b(3,5)|^{2}}{\left(w_{52}-w_{53}+w_{32}\right)}\right] \\
& \qquad+3 l\left|b_{0}\right|^{2} \tan ^{2}(\eta) \mathrm{p} / \mathrm{Lp}_{\mathrm{o}},
\end{aligned}
$$

where $\eta$ is the angle between the c-field and radiation field, and in this case, $\left|b_{0}\right|=(0.942)_{v_{0}} / l$. The latter form results when the matrix elements are evaluated for cesium through terms linear in c-field, and the Bohr frequencies are evaluated through the quadratic c-field terms.

A similar calculation for the $(4,1) \leftrightarrow(3,1)$ transition in cesium gives

$$
\delta \omega_{\text {res }} \cong l\left|\mathrm{~b}_{\mathrm{o}}\right|^{2} \tan ^{2}(\eta) \mathrm{p} / 15 \mathrm{Lp}_{\mathrm{o}} 2 \pi(7.00) 10^{5} \mathrm{H}_{\mathrm{c}} .
$$




\section{REFERENCES}

[1] F. Bloch and A.Siegert, Phys.Rev. 57, 522 (1940). See also references [3], [7], and [10].

[2] H. Salwen, Phys。Rev。99, 1274 (1955).

[3] N. Ramsey, Phys.Rev。100, 1191 (1955), and in Recent Research in Molecular Beams, I. Estermann, Ed. (Academic Press, New York, 1959).

[4] M. Mizushima, Phys。Rev. 133, A4l4 (1964). In the last section of this paper, the frequency shifts of some resonances in cesium that are interpreted by Mizushima as natural shifts are instead most likely caused by c-field nonuniformity. See reference [18].

[5] J. Shirley, Thesis (California Institute of Technology, 1963), and Phys. Rev. 138, B979 (1965).

[6] J. Winter, Ann. Phys. (Paris) $\underline{4}, 745$ (1959), S. Autler and C. Townes, Phys. Rev. 100, 703 (1955).

[7] J. Shirley, J.Appl. Phys。34, 783 (1963). See also, K. Freed, J. Chem。Phys. $\underline{43}, 1113$ (1965)。

[8] In a given problem few of the $b(j, k)$ will be zero or even small. However, by the same reasoning that justifies the two-level approximation we can neglect those matrix elements that would induce a transition with resonance frequency greatly different from the frequency of the resonance under consideration.

[9] I. Rabi, Phys.Rev. 51, 648 (1937). See also references [10] and [14].

[10] N. Ramsey, Molecular Beams (Oxford University Press, New York, 1956)。 
[11] R. Mockler, Advan. Electron. Electron Phys, 15, 1 (1961).

[12] The "Zeeman couplings", $b(2,3)$ and $b(3,4)$, induce transitions with resonance frequencies smaller than $\omega_{41}$ by a factor of $10^{5}$ or more for the c-field magnitudes of interest (see reference [8]).

[13] Of the two choices for the eigenvalue, $\lambda_{+}$or $\lambda_{-}$, one is a "proper" choice (corresponding to Salwen's proper root in [2]). The other is non-physical, leading to the possibility of an infinitely large frequency shift.

[14] H. Torrey, Phys. Rev。 59, 293 (1941)。

[15] F. Horner, T. Taylor, R. Dunsmuir, J. Lamb, and W . Jackson, J. Instn. Elect. Engrs. 93 III, 53 (1946).

[16] R. Beehler, W. Atkinson, L. Heim and C. Snider, IRE Trans. on Instr. I-11, 231 (1962).

[17] J. Shirley, "Effect of a sinusoidal excitation amplitude on the performance of an atomic beam spectrometer" (to be published).

[18] R. Harrach, "Radiation field dependent frequency shifts of atomic beam resonances" (to be published). 


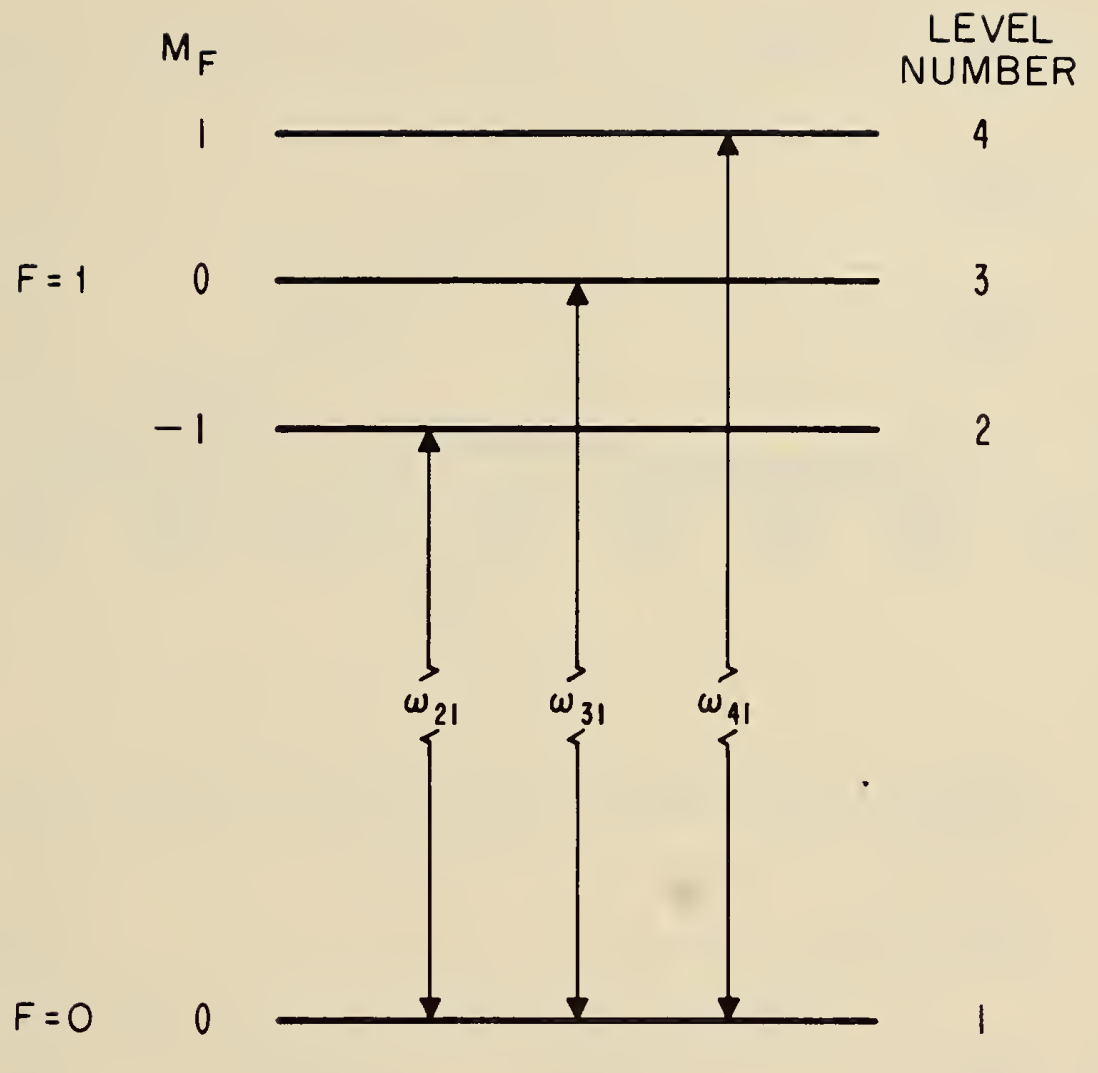

Fig. 1. Hyperfine structure levels of the ground state of a thallium-205 atom in a weak external magnetic field. 

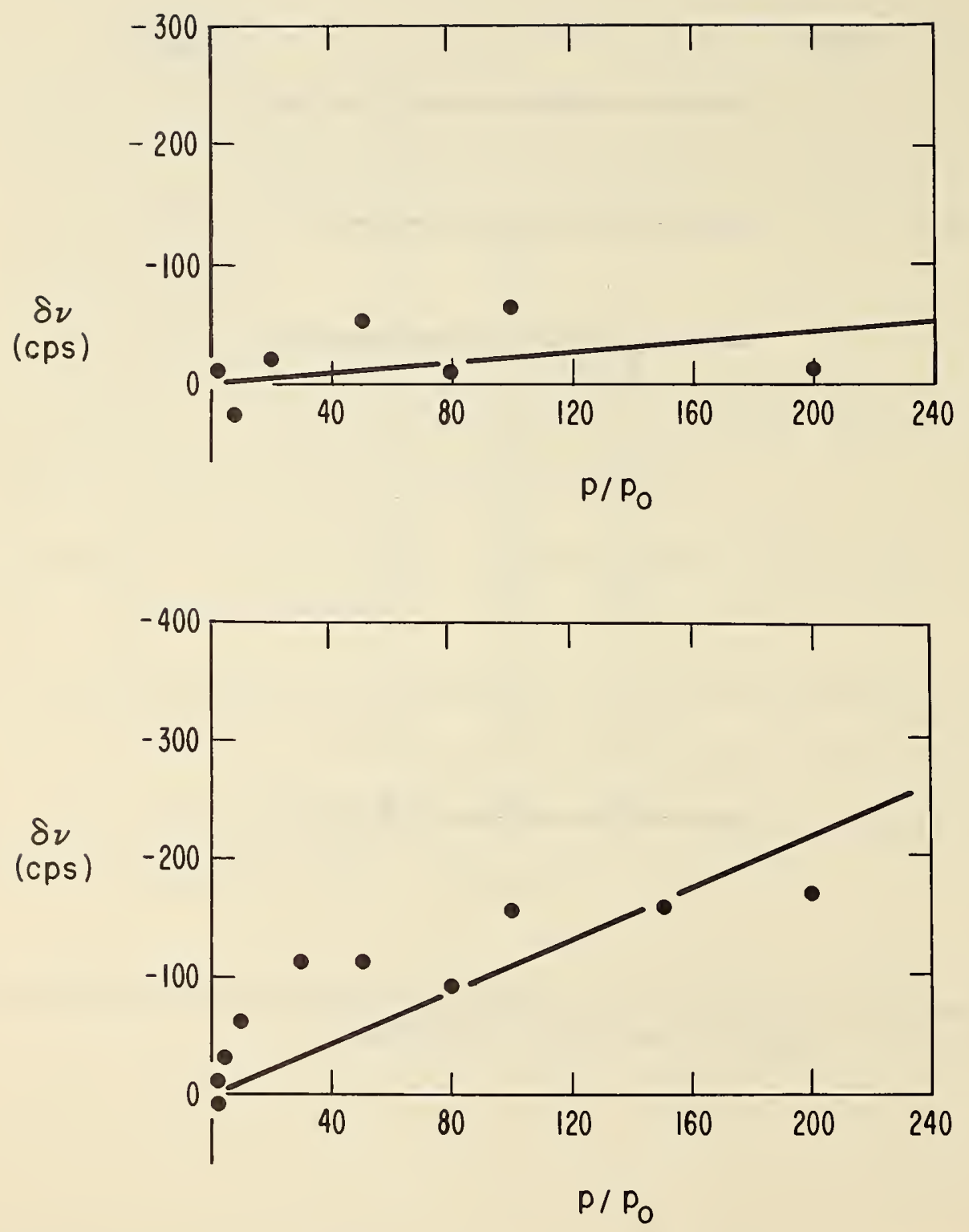

Fig. 2. Measured frequency shifts of the $(1,1) \rightarrow(0,0)$ resonance for the c-field magnitudes $\mathrm{H}_{\mathrm{c}}=0.0469$ Oe (upper) and 0.0217 Oe (lower). 

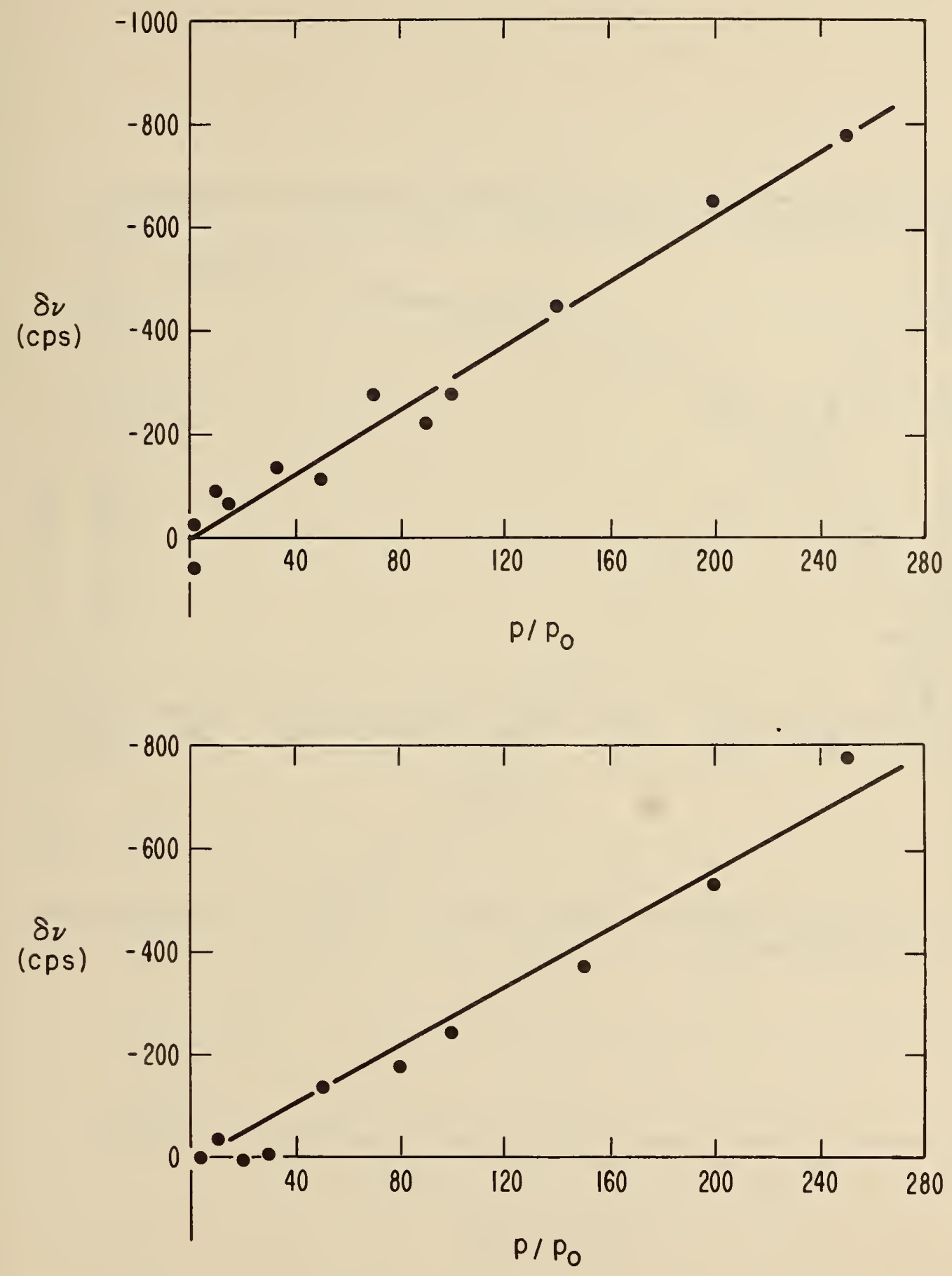

Fig. 3. Measured frequency shifts of the $(1,1) \leftrightarrow(0,0)$ resonance for $\mathrm{H}_{\mathrm{c}}=0.0141$ Oe (upper) and 0.0150 Oe (lower). 


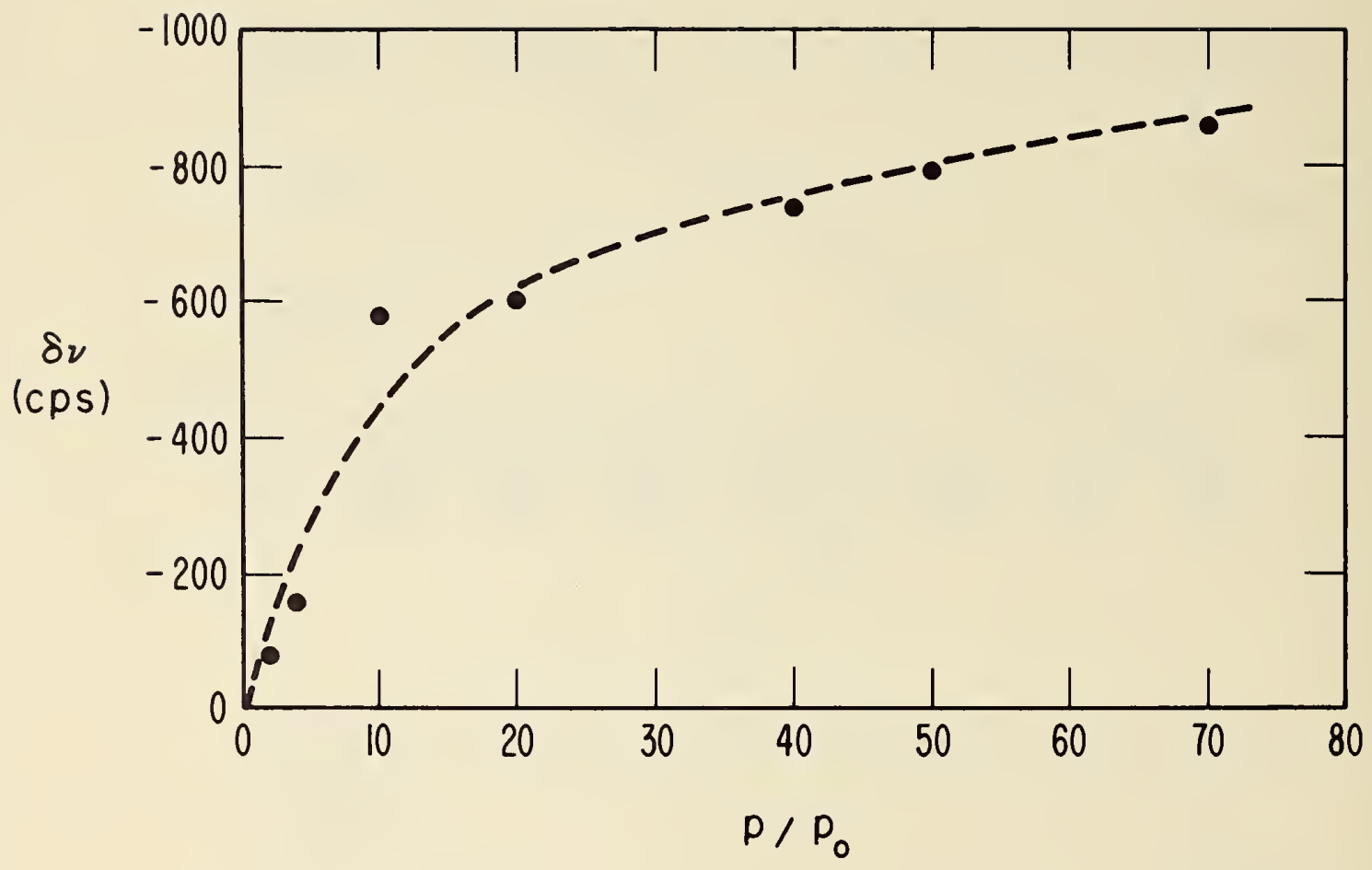

Fig. 4. Measured frequency shift of the $(1,1) \rightarrow(0,0)$ resonance for $\mathrm{H}_{\mathrm{c}}=0.0108 \mathrm{Oe}$. 


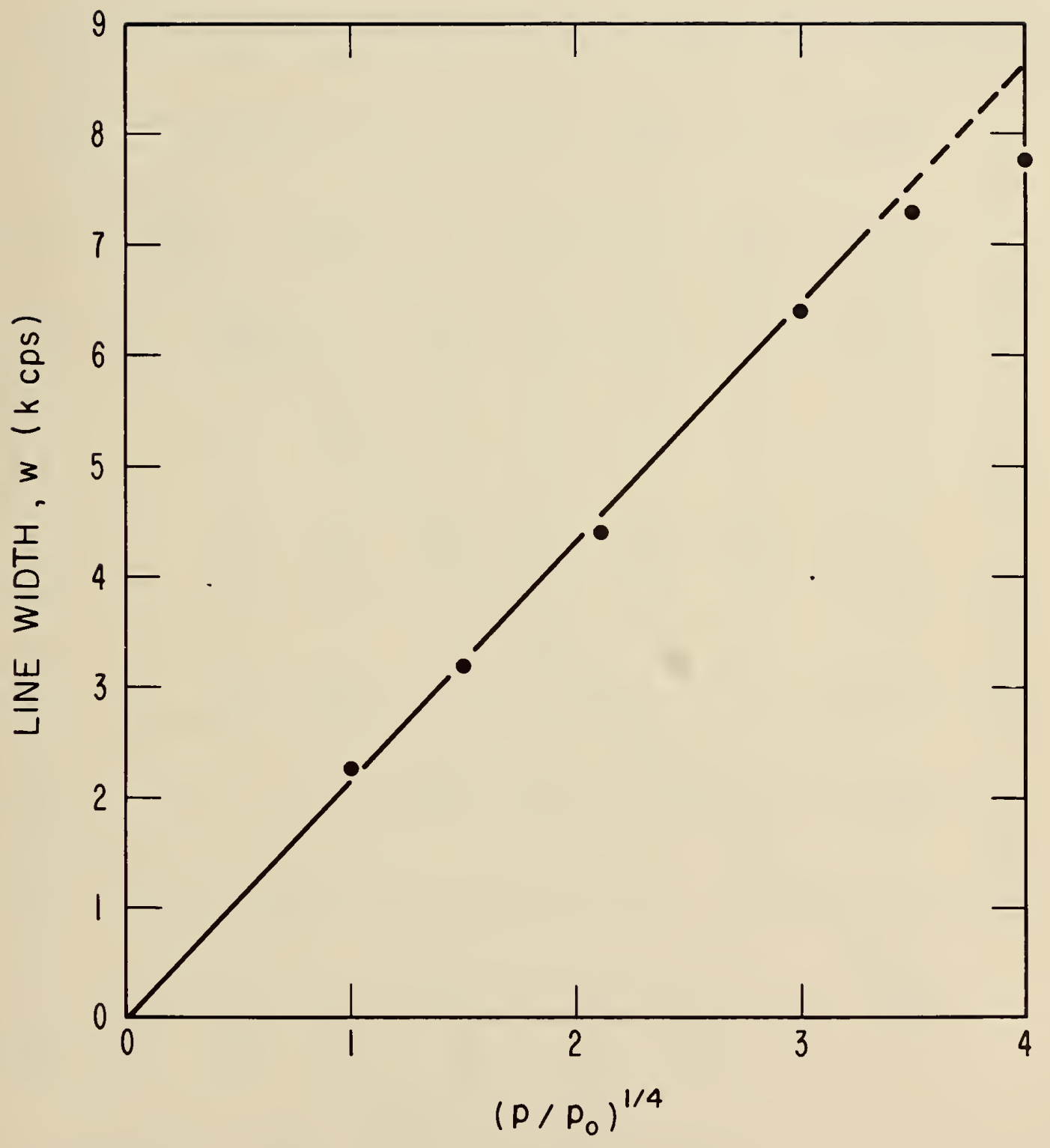

Fig. 5. Measured variation of the resonance line width as a function of excitation intensity. 


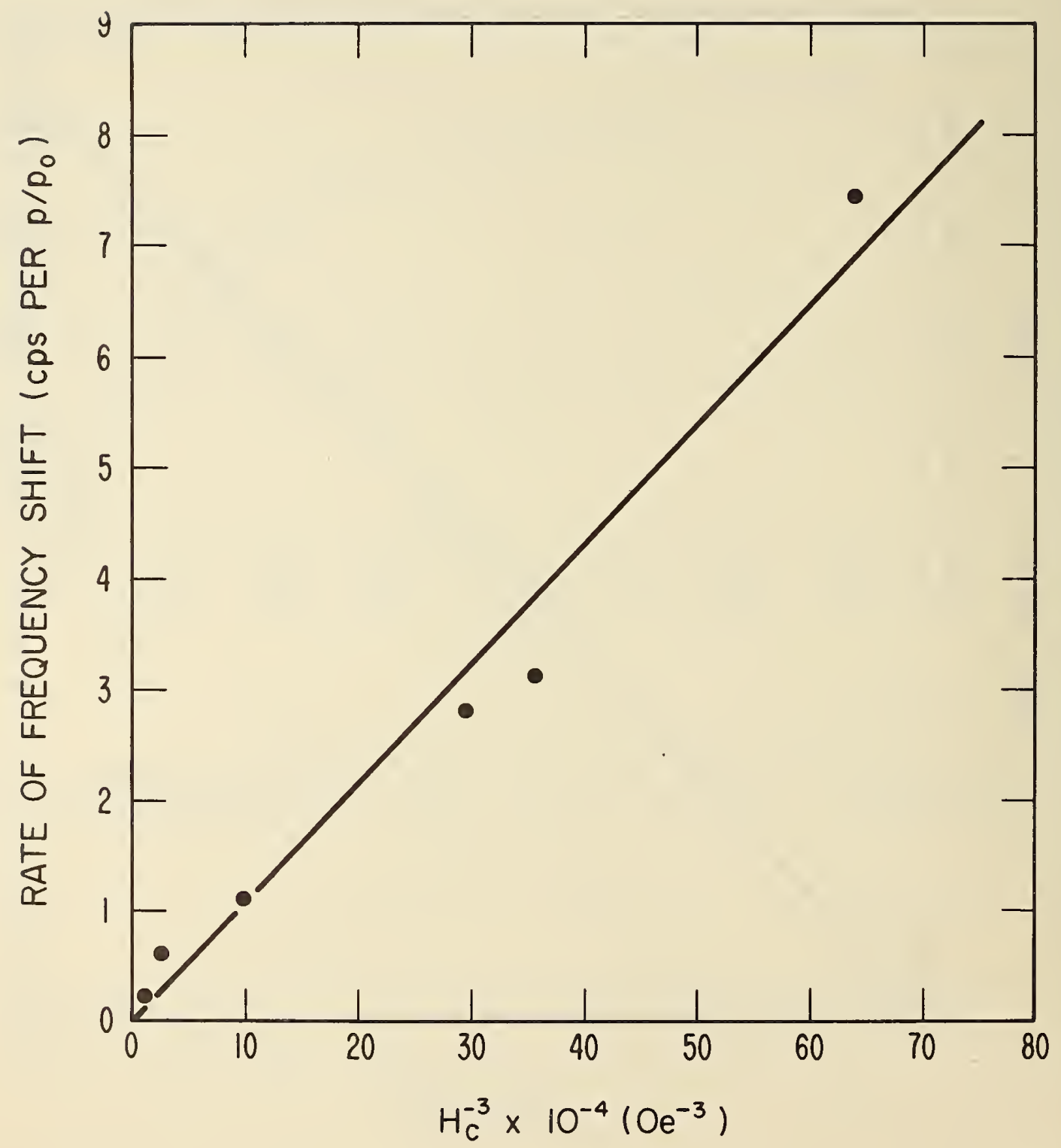

Fig. 6. Rate of frequency shift as a function of c-field magnitude. 


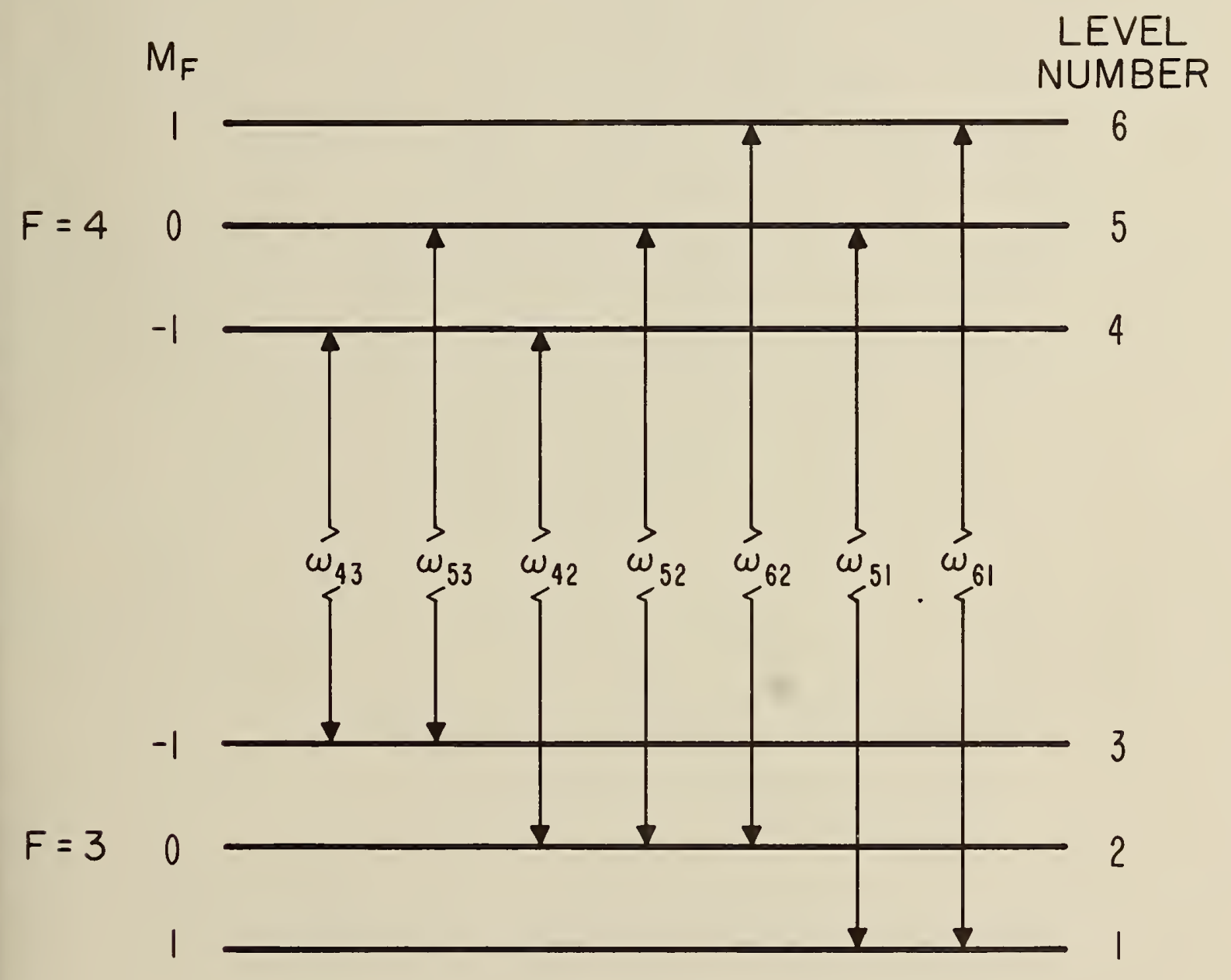

Fig. 7. Some hyperfine structure levels of the ground state of a cesium-133 atom in a weak external magnetic field. 




\section{U.S. DEPARTMENT OF COMMERCE}

WASHINGTON, D.C. 20230

OFFICIAL BUSINESS 